\title{
Symbolic generic initial systems of star configurations
}

\author{
M. Dumnicki, T. Szemberg*, J. Szpond, H. Tutaj-Gasińska
}

June 28, 2021

\begin{abstract}
The purpose of this note is to describe limiting shapes of symbolic generic initial systems of star configurations in projective spaces $\mathbb{P}^{n}$ over a field $\mathbb{K}$ of characteristic 0 .
\end{abstract}

Keywords generic initial ideals, Newton polytopes, star configurations, symbolic powers

Mathematics Subject Classification (2000) MSC 14C20 - MSC 13C05 . MSC 14N05 · MSC 14H20 · MSC 14A05

\section{Introduction}

In recent years there has been increasing interest in asymptotic invariants attached to graded families of ideals, see e.g. [17, Section 2.4.B]. For a homogeneous ideal $I$, Mayes introduces in [19] symbolic generic initial systems $\left\{\operatorname{gin}\left(I^{(m)}\right)\right\}_{m}$. Here $\operatorname{gin}(J)$ denotes the reverse lexicographic generic initial ideal of a homogeneous ideal $J$ and $J^{(m)}$ denotes the $m$ th symbolic power of $J$. For a monomial ideal $J$ one defines its Newton polytope $P(J)$. Mayes studies the limiting shape associated to $I$ as the complement in the positive octant in $\mathbb{R}^{n}$ of the asymptotic Newton polytope

$$
\Delta(I)=\bigcup_{m=1}^{\infty} \frac{1}{m} P\left(\operatorname{gin}\left(I^{(m)}\right)\right)
$$

for generic points in $\mathbb{P}^{2}$ under assuming the Segre-Harbourne-Gimigliano-Hirschowitz Conjecture, (see 2] for recent account on this conjecture). In this note we study limiting shapes for star configurations in projective spaces of arbitrary dimension. Star configurations have received much attention recently, partly because they are a nice source of interesting and computable examples, see e.g. the nice survey [11]. Our main result here is the following theorem.

Theorem 1.1. Let I be the ideal of points defined as $n$-fold intersection points of $s \geqslant n$ general hyperplanes in $\mathbb{P}^{n}$. Then $\Gamma(I)=\overline{\left(\mathbb{R}_{\geqslant 0}\right)^{n} \backslash \Delta(I)}$ is the simplex in $\mathbb{R}^{n}$ with vertices in the origin $A_{0}$ and in the points $A_{1}, \ldots, A_{n}$, where

$$
A_{i}=(\underbrace{0, \ldots, 0}_{i-1}, \frac{s-(i-1)}{n-(i-1)}, \underbrace{0, \ldots, 0}_{n-i}) .
$$

*The second named author was partially supported by NCN grant UMO-2011/01/B/ST1/04875 


\section{Generic initial ideals}

Let $S(n)=\mathbb{K}\left[x_{1}, \ldots, x_{n}\right]$ be a polynomial ring over a field $\mathbb{K}$ of characteristic 0 . Let $\succ$ be the reversed lexicographical order on monomials in $S(n)$. Recall that $\succ$ is a total ordering defined as

$$
x_{1}^{p_{1}} \cdot \ldots \cdot x_{n}^{p_{n}} \succ x_{1}^{q_{1}} \cdot \ldots \cdot x_{n}^{q_{n}}
$$

if and only if, $\sum_{i=1}^{n} p_{i}>\sum_{i=1}^{n} q_{i}$ or $\sum p_{i}=\sum q_{i}$ and there exists an index $k$ such that $p_{\ell}=q_{\ell}$ for all $\ell>k$ and $p_{k}<q_{k}$.

For a homogeneous ideal $I \subset S(n)$, its initial ideal in $(I)$ is the ideal generated by leading terms of all elements of $I$. Recall that the leading term in $(f)$ of a polynomial $f \in S$ is the greatest (with respect to the fixed order, here $\succ$ ) monomial summand of $f$. Initial ideals are of interest because they share many properties with the original ideals whereas they are easier to handle computationally, see e.g. [15].

Even better behaved are generic initial ideals. We describe now briefly how they are defined. To begin with, recall that $\operatorname{GL}(n, \mathbb{K})$ acts on $S(n)$ by the change of coordinates. The Borel subgroup $\mathbb{T}$ of $\mathrm{GL}(n, \mathbb{K})$ consists of upper triangular matrices

$$
\mathbb{T}=\left\{A \in \operatorname{GL}(n, \mathbb{K}): a_{i j}=0 \text { for all } j<i\right\} .
$$

Building upon ideas of Galligo [10, Mark Green initiated in [12] a systematic study of generic initial ideals, see also [13]. Theorem of Galligo [10] assures that for a homogeneous ideal $I$ and a generic choice of coordinates, the initial ideal in $(I)$ of $I$ is $\mathbb{T}$-fixed. In order to indicate that this property holds, we write gin $(I)$ for in $(I)$. It follows from the same theorem that gin $(I)$ is well defined, in particular uniquely determined by $I$. Generic initial ideals carry even more information on original ideal $I$ than arbitrary initial ideals do. For example Hilbert functions of $I$ and $\operatorname{gin}(I)$ are equal [13, page 90].

\section{Limiting shape of a graded family of ideals}

Following Mayes, we recall here a construction of a solid associated to a polynomial ideal with zero-dimensional support. To some extend this construction resembles that of Okounkov bodies [18, even though the resulting body in our case need not to be convex.

Let $I_{\bullet}=\left\{I_{m}\right\}$ be a graded family of ideals in $S(n)$, i.e. a collection of ideals $I_{m} \subset S(n)$ such that

$$
I_{k} \cdot I_{\ell} \subset I_{k+\ell}
$$

holds for all $k, \ell \geqslant 1$, see [17, Definition 2.4.14].

Assume that all ideals $I_{m}$ are of finite colength i.e. $\operatorname{length}\left(S(n) / I_{m}\right)$ is finite for all $m \geqslant 1$. To such a family one associates an invariant analogous to the volume of a graded linear series (this invariant is actually called a volume in [19], we prefer here however to follow the notation from [17, Definition 2.3.40]).

Definition 3.1 (Multiplicity). The multiplicity of a graded family $I_{\bullet}$ is the real number

$$
\operatorname{mult}\left(I_{\bullet}\right):=\limsup _{m \rightarrow \infty} \frac{\operatorname{length}\left(S(n) / I_{m}\right)}{m^{n} / n !} .
$$

As customary we associate to a monomial $M=x_{1}^{m_{1}} \cdot \ldots \cdot x_{n}^{m_{n}} \in S(n)$ a point $P(M)=\left(m_{1}, \ldots, m_{n}\right)$ in $\mathbb{R}^{n}$. 
Definition 3.2 (Newton polytope). Let $J$ be an arbitrary monomial ideal in $S(n)$. The Newton polytope $P(J)$ of $J$ is the convex hull of the set $\left\{P(M) \in \mathbb{R}^{n}: M \in J\right\}$.

Given a graded family $I_{\bullet}$ of monomial ideals we are interested in the limiting shape of the Newton polytopes $P\left(I_{m}\right)$ defined as

$$
\Delta\left(I_{\bullet}\right):=\bigcup_{m=1}^{\infty} \frac{1}{m} P\left(I_{m}\right) .
$$

For a monomial ideal $J \subset S(n)$, we denote by $Q(J)$ the closure of the complement of the Newton polytope $P(J)$ in the positive octant. It is well known, see e.g. [19, Proposition 2.14] and [20, Theorem 1.7 and Lemma 2.13] that if $J$ is supported on a zero-dimensional subscheme then

$$
\operatorname{mult}(J)=n ! \cdot \operatorname{vol}_{\mathbb{R}^{n}}(Q(J)) .
$$

Here $\operatorname{vol}_{\mathbb{R}^{n}}$ denotes the standard Euclidean volume on $\mathbb{R}^{n}$ normalized so that the unit cube $[0,1]^{n}$ has volume 1 .

The asymptotic counterpart of sets $Q\left(I_{m}\right)$ for a graded family $I_{\bullet}$ of ideals is defined as

$$
\Gamma\left(I_{\bullet}\right)=\bigcap_{m=1}^{\infty} \frac{1}{m} Q\left(I_{m}\right) .
$$

Of course $\Gamma\left(I_{\bullet}\right)$ is the closure of the complement of the convex set $\Delta\left(I_{\bullet}\right)$. Thus it is a coconvex body as studied in [16].

To an arbitrary ideal $J$, we associate a graded family of monomial ideals

$$
G_{m}:=\operatorname{gin}\left(J^{(m)}\right) \text {. }
$$

We write then simply $\Gamma(J)$ for $\Gamma\left(G_{\bullet}\right)$.

\section{Star configurations}

Let $s \geqslant n$ be a fixed integer and let $H_{1}, \ldots, H_{s}$ be general hyperplanes in $\mathbb{P}^{n}$. Each $n$ of these hyperplanes determine then a unique point in $\mathbb{P}^{n}$, the intersection point of all of them. Thus the set $Z$ consisting of all these points has exactly $\left(\begin{array}{l}s \\ n\end{array}\right)$ elements. Let $I=I_{Z} \subset S(n+1)$ be the homogeneous ideal associated to the reduced scheme $Z$. We consider the graded family of symbolic powers of $I$

$$
I_{m}:=I^{(m)} .
$$

Symbolic powers of ideals supported on zero-dimensional reduced schemes are saturated, see e.g. [1, Lemma 1.3]. A property of saturated ideals important for the construction carried out in the next section is the following result due to Green [12, Theorem 2.21].

Proposition 4.1 (Green). Let $J$ be a saturated ideal in $S(n+1)$. Then no minimal generator of the generic initial ideal gin $(J)$ contains the variable $x_{n+1}$.

It follows that in the above set-up of a star configuration, even though the ideals $I^{(m)}$ are contained in $S(n+1)$, their generic initial ideals $\operatorname{gin}\left(I^{(m)}\right)$ can be naturally considered as contained in $S(n)$. Thus their Newton polytopes and so also $\Delta(I)$ and $\Gamma(I)$ are naturally contained in $\mathbb{R}^{n}$.

Now we are in the position to proof Theorem 1.1 . 
Proof of Theorem 1.1. Let $H_{1}, \ldots, H_{s}$ be general hyperplanes in $\mathbb{P}^{n}$ defined as zero-sets of linear polynomials $h_{1} \ldots, h_{s}$. Let $Z$ be the set consisting of points where $n$ of these hyperplanes meet. By generality $Z$ contains exactly $\left(\begin{array}{l}s \\ n\end{array}\right)$ mutually distinct points. Let $I$ be the ideal of $Z$.

It is enough to show that points $A_{i}$ are intersection points of the coordinate axes with the sets $\Delta(I)$ and $\Gamma(I)$. Indeed, taking this for granted for a while, we show how the assertion of the Theorem follows. To this end let $W$ denote the convex hull of the set $\left\{A_{0}, A_{1}, \ldots, A_{n}\right\}$. Since $\Delta(I)$ is a convex set, its complement $\Gamma(I)$ is for sure contained in $W$. On the other hand the volume of $W$ is

$$
\operatorname{vol}_{\mathbb{R}^{n}}(W)=\frac{1}{n !} \cdot \frac{s}{n} \cdot \frac{s-1}{n-1} \cdot \ldots \cdot \frac{s-(n-1)}{1}=\frac{1}{n !}\left(\begin{array}{c}
s \\
n
\end{array}\right) .
$$

According to (11) the volume of $\Gamma(I)$ is equal to $\frac{1}{n !}$ times the number of points supporting $I$. Hence $\operatorname{vol}_{\mathbb{R}^{n}} \Gamma(I)=\operatorname{vol}_{\mathbb{R}^{n}}(W)$ and since both sets are closed and $\Gamma(I) \subset W$, they are equal.

In fact, the above argument shows a little bit more. It is namely enough to prove that $A_{1}, \ldots, A_{n}$ are contained in $\Delta(I)$. This forces $\Gamma(I)$ to be contained in $W$ and we are done again.

Turning thus to the containment of points $A_{1}, \ldots, A_{n}$ in $\Delta(I)$ by way of the warm up, we show that $A_{1}$ appears in $P\left(\operatorname{gin}\left(I^{(n)}\right)\right)$. To this end note first that the product $h_{1,2, \ldots, s}=h_{1} \cdot \ldots \cdot h_{s}$ is by construction an element of $I^{(n)}$. Since these forms are general, their product $h_{1,2, \ldots, s}$ contains the monomial $x_{1}^{s}$, which is then the leading term, so that $\operatorname{gin}\left(I^{(n)}\right)$ contains this monomial as well. Thus, after scaling, we get the point $A_{1}=\left(\frac{s}{n}, 0, \ldots, 0\right) \in \frac{1}{n} P\left(\operatorname{gin}\left(I^{(n)}\right)\right)$. We will give an additional interpretation of this vertex right after the current proof.

Claim. Now we will show that $\operatorname{gin}\left(I^{(n+1-k)}\right)$ contains the monomial $x_{n+1-k}^{s+1-k}$.

Since we work in characteristic zero and symbolic powers agree with differential powers via the Zariski-Nagata Theorem [9, Section II.3.9], the elements of degree $s+1-k$ in $I^{(n+1-k)}$ for $k=1, \ldots, n$ form a vector space. Its dimension is at least equal to $\left(\begin{array}{c}s \\ k-1\end{array}\right)$, the number of distinct products of $k-1$ forms $h_{j_{1}, \ldots, j_{k-1}}=$ $h_{j_{1}} \cdot \ldots \cdot h_{j_{k-1}}$ with $1 \leqslant j_{1}<j_{2}<\ldots<j_{k-1} \leqslant s$. Indeed, all products of the form

$$
\frac{h_{1} \cdot \ldots \cdot h_{s}}{h_{j_{1}, \ldots, j_{k-1}}}
$$

vanish in all points of $Z$ to order at least $n+1-k$.

Subclaim. These products are moreover linearly independent. This can be seen as follows. Suppose that there is a linear combination with

$$
\sum_{1 \leqslant j_{1}<\ldots<j_{k-1} \leqslant s} \lambda_{j_{1}, \ldots, j_{k-1}} \cdot \frac{h_{1} \cdot \ldots \cdot h_{s}}{h_{j_{1}, \ldots, j_{k-1}}}=0 .
$$

Restricting this sum to the intersection $Y=H_{\ell_{1}} \cap \ldots \cap H_{\ell_{k-1}}$ we obtain

$$
\lambda_{\ell_{1}, \ldots, \ell_{k-1}} \cdot h_{i_{1}, \ldots, i_{s+1-k}}=0
$$

for $\left\{i_{1}, \ldots, i_{s+1-k}\right\}=\{1,2, \ldots, s\} \backslash\left\{\ell_{1}, \ldots, \ell_{k-1}\right\}$. Since none of the forms $h_{i_{1}}, \ldots, h_{i_{s+1-k}}$ vanishes identically on $Y$, it must be $\lambda_{\ell_{1}, \ldots, \ell_{k-1}}=0$. This argument works for arbitrary pick of $\ell_{1}, \ldots, \ell_{k-1}$ so that we can conclude that all $\lambda_{i_{1}, \ldots, i_{k-1}}$ vanish. This establishes the Subclaim.

It is easy to see that there are $\left(\begin{array}{c}s \\ k-1\end{array}\right)-1$ monomials of degree $s+1-k$ which are strictly bigger than $x_{n+1-k}^{s+1-k}$ with respect to the order $\succ$. Hence there exists a 
non-zero linear combination $f$ of forms of degree $s+1-k$ as in (2), with vanishing coefficients at all monomials strictly bigger than $x_{n+1-k}^{s+1-k}$ with respect to the order $\succ$. By the generality of the forms, the coefficient of $f$ at $x_{n+1-k}^{s+1-k}$ does not vanish. Hence $x_{n+1-k}^{s+1-k}$ is the leading monomial of $f$ and thus it appears in $\operatorname{gin}\left(I^{n+1-k}\right)$. This finishes the proof of Theorem 1.1 .

\section{Some asymptotic invariants attached to a homogeneous ideal}

\section{1 $A_{1}$ and Waldschmidt constants}

Now we give an interpretation of vertices $A_{1}$ and $A_{n}$. We recall first a couple of definitions.

Definition 5.1 (Initial degree). Let $I$ be a homogeneous ideal in a polynomial ring. Then

$$
\text { the initial degree } \alpha(I)
$$

is defined as the minimal number $t$ such that there exists a non-zero element of degree $t$ in $I$.

The asymptotic counter-part of the initial ideal is called Waldschmidt constant of $I$. It has been rediscovered recently and intensively studied by Harbourne, see e.g. [5] and [14].

Definition 5.2 (Waldschmidt constant). The Waldschmidt constant of $I$ is defined as

$$
\widehat{\alpha}(I):=\lim _{m \rightarrow \infty} \frac{\alpha\left(I^{(m)}\right)}{m} .
$$

The existence of the limit in the definition above follows from the sub-additivity of initial degrees. This also implies in a standard way that the Waldschmidt constant is in fact the infimum of the quotients $\frac{\alpha\left(I^{(m)}\right)}{m}$.

Waldschmidt constants for points forming a star configuration of $n$-wise intersection points of $s \geqslant n$ general hyperplanes in $\mathbb{P}^{n}$ was computed in [3, Example 8.3.4]. We have

$$
\widehat{\alpha}(I)=\frac{s}{n}
$$

in this case. This corresponds to the non-zero coordinate of the vertex $A_{1}$.

Remark 5.3. In fact it is easy to see that $\alpha\left(I^{(m)}\right)$ is the minimal coordinate of a point in $P\left(\operatorname{gin}\left(I^{(m)}\right)\right)$ sitting on the $x_{1}$-axis. This follows from the Borel invariance of generic initial ideals. Indeed if $f$ is a monomial in $\operatorname{gin}\left(I^{(m)}\right)$ divisible by some $x_{i}$, then also $x_{1} \cdot \frac{f}{x_{i}}$ is contained in $\operatorname{gin}\left(I^{(m)}\right)$. This implies that the minimal degree of a non-zero element in $I^{(m)}$ (which is of course equal to the degree of its leading term) can be read of the $x_{1}$-axis.

\section{2 $A_{n}$ and asymptotic regularity}

Now we want to interpret the last vertex $A_{n}$. We begin by recalling the notion of the Castelnuovo-Mumford regularity which is one of fundamental invariants determining the complexity of an ideal, see [9, Section 20.5] and [17, Section 1.8]. 
Definition 5.4 (Castelnuovo-Mumford regularity). Let $J$ be a homogeneous ideal in a polynomial ring $S$ and let

$$
0 \rightarrow \ldots \rightarrow \bigoplus_{j} S\left(-a_{i j}\right) \rightarrow \ldots \bigoplus_{j} S\left(-a_{1 j}\right) \rightarrow \bigoplus_{j} S\left(-a_{0 j}\right) \rightarrow J \rightarrow 0
$$

be a minimal free resolution of $I$. Then the Castelnuovo-Mumford regularity of $J$ is the non-negative integer

$$
\operatorname{reg}(J):=\max _{i, j}\left\{a_{i j}-i\right\} .
$$

In other words regularity of an ideal is governed by the maximal degree of generators of its syzygy modules. In particular we always have $\operatorname{reg}(J) \geqslant d(J)$, where $d(J)$ denotes the maximal degree in a minimal set of generators of $J$. For a Borel fixed ideal, Bayer and Stillman show in [4, Proposition 2.9] that one has actually the equality

$$
\operatorname{reg}(J)=d(J) \text {. }
$$

Since the regularity is upper-semicontinuous in flat families one has always $\operatorname{reg}(J) \leqslant$ $\operatorname{reg}(\operatorname{in}(J))$ and $\operatorname{reg}(J)=\operatorname{reg}(\operatorname{gin}(J))$ by [4, Proposition 2.11]. Now, the argument used in Remark 5.3 implies that $d(J)$ is detected by the generator of $J$ whose initial monomial is a power of $x_{n}$.

Asymptotic regularity was studied by Cutkosky, Herzog and Trung in [7]. It is defined as the real number

$$
\operatorname{areg}(J)=\lim _{m \rightarrow \infty} \frac{\operatorname{reg}\left(J^{m}\right)}{m} .
$$

As remarked in [7, Theorem 1.1] it is equal to $\lim _{m \rightarrow \infty} \frac{d\left(J^{m}\right)}{m}$.

Motivated more by geometry Cutkosky, Ein and Lazarsfeld study in [6] a new invariant associated to an ideal sheaf. Their definition works for ideal sheaves on arbitrary projective varieties, we prefer however to restrict our attention here to the projective space setting.

Definition 5.5 (the $s$-invariant). Let $\mathcal{J}$ be an ideal sheaf in $\mathcal{O}_{\mathbb{P}}$ and let $\pi: X \rightarrow \mathbb{P}^{n}$ be the blow up of $\mathcal{J}$ with exceptional divisor $F$. The $s$-invariant of $\mathcal{J}$ is defined as

$$
s(\mathcal{J}):=\min \left\{t \in \mathbb{R}: t \cdot \pi^{*}\left(\mathcal{O}_{\mathbb{P}^{n}}(1)\right)-F \text { is nef }\right\} .
$$

If $\mathcal{J}$ is the sheafification of a homogeneous ideal $I$, then we have

$$
\operatorname{areg}(I)=s(\mathcal{J}),
$$

see [17, Theorem 5.4.22].

Asymptotic regularity of symbolic powers was studied recently by Cutkosky and Kurano in [8]. It is defined as

$$
\operatorname{asreg}(I):=\lim _{m \rightarrow \infty} \frac{\operatorname{reg}\left(I^{(m)}\right)}{m} .
$$

It follows immediately from [8, Theorem 4.6] that also

$$
\operatorname{asreg}(I)=s(\mathcal{J}) .
$$

Since generic initial ideal gin $(I)$ of a homogeneous ideal $I$ in $S(n+1)$ contains among generators a power of $x_{n}$, see [19, Corollary 2.9], combining (3) with (4) and (51) we get that the non-zero coordinate of $A_{n}$ equals

$$
\operatorname{areg}(I)=\operatorname{asreg}(I)=s(\mathcal{J}) .
$$


Remark 5.6. It would be interesting to know if the remaining vertices $A_{2}, \ldots, A_{n-1}$ can be interpreted along similar lines.

We conclude this note with an example showing that one cannot reverse Theorem 1.1, i.e. the shape of $\Gamma(I)$ does not determine $I$ to be the ideal of a star configuration of points.

Example 5.7 (Points with star configuration like limiting shape). Let $I$ be the ideal of intersection points $P_{1}, \ldots, P_{6}$ of a smooth conic $C$ with a general cubic curve $D$. Then obviously $\widehat{\alpha}(I)=2$ and $\operatorname{areg}(I)=3$. Hence $\Gamma(I)$ is the triangle with vertices $A_{0}=(0,0), A_{1}=(2,0)$ and $A_{2}=(0,3)$ but the points $P_{1}, \ldots, P_{6}$ do not form a star configuration.

\section{References}

[1] Arsie, A., Vatne, J. E.: A note on symbolic and ordinary powers of homogeneous ideals, Ann. Univ. Ferrara 49 (2003), 19-30

[2] Bauer, Th., Bocci, C., Cooper, S., Di Rocco, S., Dumnicki, M., Harbourne, B., Jabbusch, K., Knutsen, A. L., Küronya, A., Miranda, R., Roe, J., Schenck, H., Szemberg, T., Teitler, Z.: Recent developments and open problems in linear series, Contributions to Algebraic Geometry, in: Piotr Pragacz (Ed.), IMPANGA Lecture Notes, European Mathematical Society Publishing House, 2012, pp. 93-140. EMS Series of Congress Reports.

[3] Bauer, Th., Di Rocco, S., Harbourne, B., Kapustka, M., Knutsen, A. L., Syzdek, W., Szemberg T.: A primer on Seshadri constants, Interactions of Classical and Numerical Algebraic Geometry, Proceedings of a conference in honor of A. J. Sommese, held at Notre Dame, May 22-24 2008. Contemporary Mathematics vol. 496, 2009, eds. D. J. Bates, G-M. Besana, S. Di Rocco, and C. W. Wampler, 362 pp.

[4] Bayer, D., Stillman, M.: A criterion for detecting $m$-regularity, Invent. Math. 87 (1987), 1-11

[5] Bocci, C., Harbourne, B.: The resurgence of ideals of points and the containment problem, Proc. Amer. Math. Soc., 138 (2010), 1175-1190

[6] Cutkosky, S. D., Ein, L., Lazarsfeld, R.: Positivity and complexity of ideal sheaves, Math. Ann. 321 (2001), 213-234

[7] Cutkosky, S. D., Herzog, J., Trung, N. V.: Asymptotic behaviour of the Castelnuovo-Mumford regularity. Compositio Math. 118 (1999), 243-261

[8] Cutkosky, S. D., Kurano, K.: Asymptotic regularity of powers of ideals of points in a weighted projective plane, Kyoto J. Math.,51 (2011), 25-45

[9] Eisenbud, D.: Commutative algebra. With a view toward algebraic geometry. Springer-Verlag, New York, 1995.

[10] Galligo, A.: A propos du théorème de préparation de Weierstrass. Fonctions de plusieurs variables complexes, pp. 543-579. Lecture Notes in Math., Vol. 409, Springer, Berlin, 1974

[11] Geramita, A. V., Harbourne, B., Migliore, J.: Star configurations in $\mathbb{P}^{n}$, J. Algebra 376 (2013), 279-299

[12] Green, M. L.: Generic initial ideals. Six lectures on commutative algebra, pp. 119-186. Progr. Math., 166, Birkhäuser Verlag, Basel, 1998

[13] Green, M. L., Stillman, M.: A tutorial on generic initial ideals. Gröbner bases and applications (Linz, 1998), pp. 90-108, London Math. Soc. Lecture Note Ser., 251, Cambridge Univ. Press, Cambridge, 1998

[14] Guardo E., Harbourne B., Van Tuyl A.: Asymptotic resurgences for ideals of positive dimensional subschemes of projective space, Adv. Math. 246 (2013), 114-127 
[15] Herzog, J., Hibi, T.: Monomial ideals. Graduate Texts in Mathematics, 260. Springer-Verlag London, Ltd., London, 2011

[16] Khovanskii, A., Timorin, V.: On the theory of coconvex bodies. arXiv:1308.1781

[17] Lazarsfeld, R.: Positivity in Algebraic Geometry. I.-II. Ergebnisse der Mathematik und ihrer Grenzgebiete, Vols. 48-49., Springer Verlag, Berlin, 2004.

[18] Lazarsfeld, R., Mustaţă, M.: Convex bodies associated to linear series, Ann. Scient. Éc. Norm. Sup., 4 série, t. 42, (2009), 783-835.

[19] Mayes, S.: The asymptotic behaviour of symbolic generic initial systems of generic points, J. Pure Appl. Alg. 218 (2014), 381-390

[20] Mustaţă, M.: On multiplicities of graded sequences of ideals, J. Algebra 256 (2002), 229-249

Marcin Dumnicki, Jagiellonian University, Institute of Mathematics, Lojasiewicza 6, PL-30-348 Kraków, Poland

E-mail address: Marcin.Dumnicki@im.uj.edu.pl

Tomasz Szemberg, Instytut Matematyki UP, Podchorążych 2, PL-30-084 Kraków, Poland. E-mail address: szemberg@up.krakow.pl

Justyna Szpond, Instytut Matematyki UP, Podchorążych 2, PL-30-084 Kraków, Poland. E-mail address: szpond@up.krakow.pl

Halszka Tutaj-Gasińska, Jagiellonian University, Institute of Mathematics, Łojasiewicza 6, PL-30-348 Kraków, Poland

E-mail address: Halszka.Tutaj@im.uj.edu.pl 